\title{
Entanglement between nitrogen vacancy spins in diamond controlled by a nano mechanical resonator
}

\author{
L. Chotorlishvili ${ }^{1}$, D. Sander ${ }^{2}$, A. Sukhov ${ }^{1}$, V. Dugaev ${ }^{1,3}$, V. R. Vieira ${ }^{4}$, A. Komnik ${ }^{5}$, J. Berakdar ${ }^{1}$ \\ ${ }^{1}$ Institut für Physik, Martin-Luther Universität Halle-Wittenberg, D-06120 Halle/Saale, Germany \\ ${ }^{2}$ Max Planck Institute of Microstructure Physics, D-06120 Halle/Saale, Germany \\ ${ }^{3}$ Department of Physics, Rzeszów University of Technology Al. Powstanców Warszawy 6, 35-959 Rzeszów, Poland \\ ${ }^{4}$ Department of Physics and CFIF, Instituto Superior Técnico, \\ Universidade Técnica de Lisboa, Avenida Rovisco Pais, 1049-001 Lisboa, Portugal \\ ${ }^{5}$ Institut für Theoretische Physik, Universität Heidelberg, Philosophenweg 19, D-69120
}

(Dated: August 1, 2018)

\begin{abstract}
We suggest a new type of nano-electromechanical resonator, the functionality of which is based on a magnetic field induced deflection of an appropriate cantilever that oscillates between nitrogen vacancy (NV) spins in daimond. Specifically, we consider a $\mathrm{Si}(100)$ cantilever coated with a thin magnetic Ni film. Magnetoelastic stress and magnetic-field induced torque are utilized to induce a controlled cantilever deflection. It is shown that, depending on the value of the system parameters, the induced asymmetry of the cantilever deflection substantially modifies the characteristics of the system. In particular, the coupling strength between the NV spins and the degree of entanglement can be controlled through magnetoelastic stress and magnetic-field induced torque effects. Our theoretical proposal can be implemented experimentally with the potential of increasing several times the coupling strength between the NV spins as compared to the maximal coupling strength reported before in P. Rabl, et al. Phys. Rev. B 79, 041302(R) (2009).
\end{abstract}

\section{INTRODUCTION}

Nano-electromechanical resonators (NEMs) are attracting intense research efforts due to a number of favorable properties such as the high sensitivity and the swift response to an external force with a low power consumption. This makes NEMs attractive for applications, e.g. for microwave switches, nano-mechanical memory elements, and for single molecule sensing $1-\underline{\underline{3}}$. The role of quantized mechanical motion coupled to other quantum degrees of freedom as well as the influence of dissipation and noise are important issues in NEMs research with numerous findings and demonstrations of applications, e.g. Refs $\stackrel{4-23}{-2}$ and further references therein. These studies also evidence the potential of NEMs for studying fundamental questions concerning the quantum-classical interrelation and issues related to entanglement and quantum correlations. Particularly interesting for the present work are spin states in nitrogen vacancy (NV) impurities in diamond ${ }^{24-31}$ as utilized for quantum information studies. Their quantum mechanical properties can be mapped onto effective two-level systems which possess very long decoherence times even at a room temperature. On the other hand, they allow for a high degree of tunability via external magnetic fields which renders possible the use of NV impurity spins as sensors. For instance, a detection of a single electronic spin by a classical cantilever was demonstrated $\operatorname{in}^{27}$. Magnetic tips attached on the free end of a cantilever generate a magnetic field gradient during oscillations, which induces a magnetic coupling between the nanomechanical oscillator and the spin system. Using the backaction due to this coupling one can readout the cantilever motion 24,25 .

One further fascinating application for the $\mathrm{NV}$ spin based nano-electromechanical resonator system is mag- netic resonance force microscopy (MRFM) which was proposed to improve the detection resolution for the three-dimensional imaging of macromolecules 27 . Operation of the MRFM is based on the detection of the magnetic force between a ferromagnetic tip and spins. The possibility to achieve strong (up to $0.1 \mathrm{MHz}$ or even stronger) coupling between the quantized motion of the nano-resonator and NV impurity spin was demonstrated in Ref. ${ }^{29}$. However, an efficient control of the coupling strength and the development of ultrasensitive cantileverbased force sensors is still a fundamental challenge. Yet another application is based on using such a resonator in order to produce controllable entanglement between spins. Despite rather long decoherence time scales it has not been demonstrated yet, to our knowledge.

In this paper we propose a new type of a nanoelectromechanical resonator system, the functionality of which is based on the NV spin controlled by a magnetic field. The nano-resonator is a cantilever, which is covered with a magnetic film, such that we can exploit magnetoelastic stress or magnetic torque effects for a controllable cantilever deflection. At the free end of the cantilever magnetic tips are mounted, as indicated in Fig. 1. The magnetic field allows for a full control of the cantilever configuration via the amplitude of the applied magnetic field. This is the major novelty of the current proposal. As will be demonstrated below, the field-induced deflection of the cantilever has a major impact on the interaction strength between the NV spins and the magnetic tips, as well as on the strength of the indirect interaction between the spins mediated by the cantilever. We will show that the asymmetry of the nanomechanical system with respect to the shape of the cantilever controlled by magnetic fields drastically changes the degree of entanglement. 
The paper is organized as follows. In the next section we introduce our system as well as the model Hamiltonian and discuss the relevant experimental parameters. Section [II]describes the magnetic field induced cantilever deflection. The subsequent Section IV focuses on the effects of the induced indirect interaction of the impurities mediated by the cantilever coupling. The influence of the asymmetry coupling on the degree of entanglement is considered in Section V] Finally, Section VI summarizes the results gives some perspectives for further progress.

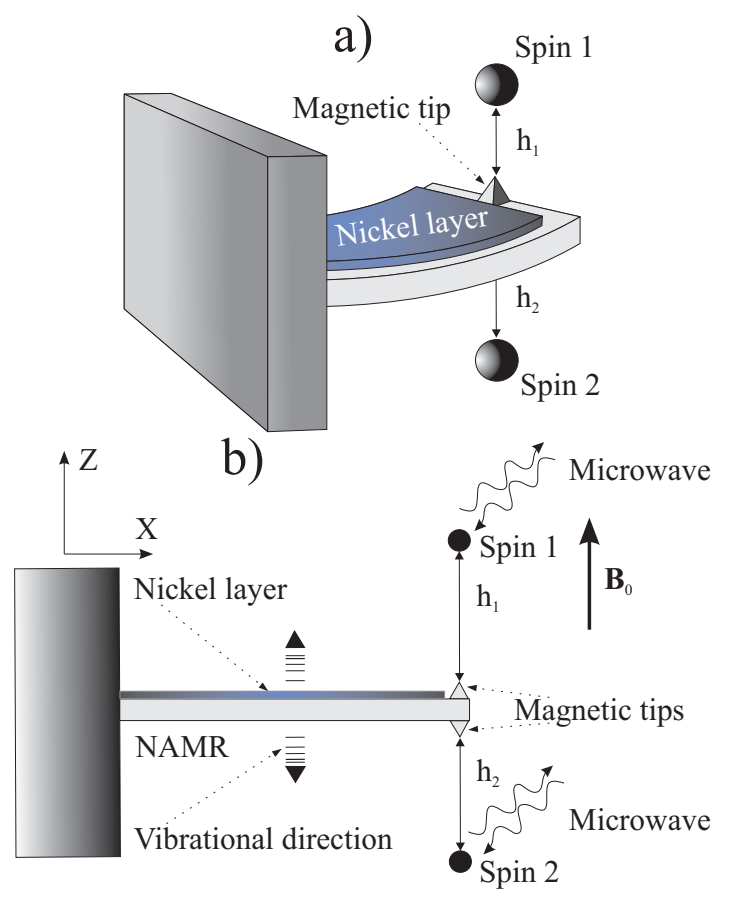

FIG. 1. Schematics of the nanomechanical resonator system discussed in the present project. a) The distances $h_{1,2}$ between the NV spins and magnetic tips can be adjusted by the field-induced deflection of the cantilever. The deflection is controlled by the applied magnetic field $B_{0}$. In the absence of the external constant magnetic field $B_{0}=0$ the system becomes symmetric, and $\left.h_{1}=h_{2}=h_{0} \approx 25 \mathrm{~nm} \mathrm{~b}\right)$. Microwaves are used to drive spin transitions at the NV centers of spin 1 and spin 2. b) Two magnetic tips are mounted on the free end of the cantilever, which mechanically oscillates in the z-axis direction. The length of the cantilever is of the order $L \sim 3000 \mathrm{~nm}$. The cantilever surface is covered with a $10 \mathrm{~nm}$ thin Ni film. See Section ЏII for further details.

\section{THEORETICAL MODEL}

The system of our interest is schematically shown in Fig. 1 Magnetic tips are attached to the free ends of the cantilever on both sides. The deflection of the cantilever and consequently the distance between magnetic tips and spins $h_{1,2}$ is controlled via the magnetic field $\vec{B}_{0}$ applied along the $Z$ axis. The nitrogen vacancy center in diamond consists of a substitutional nitrogen atom with

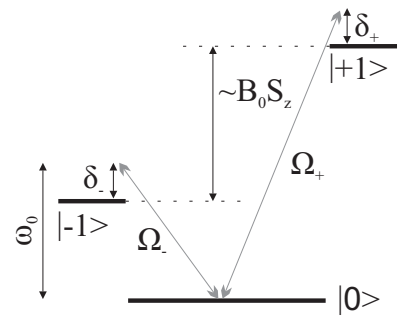

FIG. 2. Configuration of the energy levels. $\Omega_{ \pm}$stand for the Rabi frequencies between the ground and the excited levels $(|0>|-1>$,$) and (|0>| 1>,) . \delta_{ \pm}$denote the detuning between microwave frequency $\omega_{0}$ and transition frequencies.

an adjacent vacancy. The total spin of the many-electron orbital ground state of the NV center is described by the spin triplet $S=1, m_{\mathrm{S}}=-1,0,1$. States with different $\left|m_{\mathrm{S}}\right|$ are separated by a zero field splitting barrier ${ }^{24}$ which is of the order $\omega_{0}=2.88 \mathrm{GHz}$. This splitting is an intrinsic property of the NV spin system and originates ${ }^{31}$ from the effect of the spin-orbit and spin-spin interactions leading to the single-axis spin anisotropy $D S_{\mathrm{z}}^{2} \approx \hbar \omega_{0}$. In the theoretical description we set $\hbar=1$. The role of the applied external magnetic field is twofold: First of all, the applied external magnetic field $\mu_{\mathrm{B}} B_{0}<\hbar \omega_{0}$, due to the Zeeman shift proportional to $B_{0} S_{\mathrm{z}}$, removes the degeneracy of the levels $|-1>| 1>$,. Besides, as will be shown below, the external magnetic field, due to the thin magnetic Ni film deposited on the cantilever, modifies the shape of the cantilever. We will demonstrate that the asymmetry of the cantilever position between spins 1 and 2 (Fig. 1), induced by an external magnetic field, has important consequences for the coupling strength and the entanglement between the NV spins. Therefore, one can control the degree of entanglement and increase the interaction strength between the NV spins. This constitutes an important advance in nanomechanics.

The Hamiltonian of the single NV spin system reads $\underline{24}$

$$
H_{\mathrm{NV}}=\sum_{i= \pm 1}\left(-\delta_{i}|i\rangle\langle i|+\frac{\Omega_{i}}{2}(|0\rangle\langle i|+| i\rangle\langle 0|)\right) .
$$

In the case of a weak magnetic field $\mu_{\mathrm{B}} B_{0} \ll \hbar \omega_{0}$ $(\ll 30 \mathrm{mT})$ one can neglect level splitting and set $\delta_{-}=$ $\delta_{+}, \quad \Omega_{-}=\Omega_{+}$. In this case the Hamiltonian (1) couples the ground state $\mid 0>$ to the "bright" superposition of the excited states $\mid b>=\frac{1}{\sqrt{2}}(|-1>+| 1>)$, while the "dark" state $\mid d>=\frac{1}{\sqrt{2}}(|-1>-| 1>)$ is readout and decoupled from the process. Since only two states are involved, the NV spin triplet can be described via a $S=1 / 2$ pseudo-spin model. If the external constant magnetic field is strong enough, the splitting between the levels $|-1>| 1>$, is larger and the system becomes identical to the three level generalized Jaynes-Cummings model in the so called lambda configuration $\underline{32,33}$ (See Fig. 21. 
A peculiarity of the generalized Jaynes-Cummings model is the expectation of two different transition frequencies between states $(|0>|-1>$,$) and (|0>| 1>$,$) . The$ Hamiltonian of the system holds a $S U(3)$ symmetry and can be specified in terms of the Gell-Mann generators ${ }^{32}$. The three-level Jaynes-Cummings model is exactly solvable in the general case. If the rf-field contains only one frequency resonant to the transition $(|0>|-1>$, the system can be reduced to the $S=1 / 2$ pseudo-spin model which will describe transitions between the states $|-1>| 0>$, only, since the transition between the levels $|1>| 0>$, is off resonance and therefore forbidden. However, the Rabi frequency $\Omega_{-}$of the transition between the states $|-1>| 0>$, in this case is different from the transition frequency between the ground state $\mid 0>$ and the bright superposition of the excited states $\mid b>=\frac{1}{\sqrt{2}}(|-1>+| 1>)$. Nevertheless, in both cases our system can be described via the effective two-level model with a different Rabi transition frequency. The Hamiltonian of the system in the frame rotating with the frequency of the rf-field has the form

$$
H_{\mathrm{S}}=-\delta|-1\rangle\langle-1|+\frac{\Omega(B)}{2}(|-1\rangle\langle 0|+| 0\rangle\langle-1|) .
$$

Here $\delta=\omega_{0}-\Omega(B)$ is the detuning between the microwave frequency and the intrinsic frequency of the spins. The Rabi frequency of the transition between up and down spin states $\Omega(B)$ depends on the amplitude of the magnetic field and, thus, includes both limiting cases. In the case of zero external constant magnetic field $B=0$ and zero splitting between the levels $|-1>| 1>$, the Rabi frequency for the transition between the bright $\mid b>$ and the ground $\mid 0>$ states is equal to $\Omega(0)=\Omega_{0}$, while in the case of a nonzero external field and nonzero splitting for the transition between levels $|-1>| 0>$, the Rabi frequency is equal to:

$$
\Omega(B)=\Omega_{0}-\Delta \Omega(B), \quad \Delta \Omega(B)=\mu_{\mathrm{B}}\left(B_{0}+B_{\mathrm{ms}}\right) .
$$

Here $B_{0}$ is the amplitude of the external constant magnetic field applied on the system, $B_{\mathrm{ms}}$ is the magnetic field produced by the magnetic film on the cantilever at the position of the spins Spin 1 and 2, and it is $B_{0}>B_{\mathrm{ms}}$. The eigenbasis of the Hamiltonian (2) is given by the following states 25

$$
\begin{aligned}
& |g\rangle=\cos (\theta / 2)|-1\rangle+\sin (\theta / 2)|0\rangle, \\
& |e\rangle=-\sin (\theta / 2)|-1\rangle+\cos (\theta / 2)|0\rangle, \\
& \tan \theta=-\frac{\Omega}{\delta} .
\end{aligned}
$$

In the basis (4) the components of the pseudo-spin operator have the form

$$
\sigma_{z}=|e\rangle\langle e|-| g\rangle\left\langle g\left|, \quad \sigma_{+}=\right| e\right\rangle\left\langle g\left|, \quad \sigma_{-}=\right| g\right\rangle\langle e|
$$

while the $Z$ component of the spin $S_{\mathrm{z}}=\frac{1}{2}(|0><0|-\mid-$ $1><-1 \mid$ ) and the Hamiltonian of the system $H_{\mathrm{S}}$ (given by eq. (2) ) reads

$$
\begin{aligned}
& S_{\mathrm{z}}=\frac{1}{2}\left[\cos \theta \sigma_{\mathrm{z}}+\sin \theta\left(\sigma_{+}+\sigma_{-}\right)\right], \\
& H_{\mathrm{S}}=\frac{1}{2} \omega \sigma_{\mathrm{z}}, \quad \omega=\left(\Omega^{2}+\delta^{2}\right)^{1 / 2} .
\end{aligned}
$$

Taking into account eqs. (44)-(6) we have for the Hamiltonian of a single NV spin interacting with the cantilever

$$
\begin{array}{r}
\hat{H}=\frac{1}{2} \omega \sigma^{z}+\omega_{r}\left(a^{+} a+1 / 2\right)+\frac{\lambda}{2}\left(a^{+}+a\right) \times \\
{\left[\cos \theta \sigma^{z}+\sin \theta\left(\sigma^{+}+\sigma^{-}\right)\right] .}
\end{array}
$$

Now we generalize the model given by eq. (7) for the system of two spins (see Fig. 1). In the absence of an applied magnetic field the system is symmetric and the distance between the spins and the cantilever is equal $h_{1}=h_{2}=h$. However, due to the magnetic field induced cantilever deflection, the external magnetic field leads to an asymmetry $h_{1} \neq h_{2}$. The imposed asymme$\operatorname{try} \Delta h\left(B_{0}\right)=2\left|h_{1}-h_{2}\right| /\left(h_{1}+h_{2}\right), 0<\Delta h\left(B_{0}\right)<1$ can be quantified in terms of the amplitude of applied magnetic field $B_{0}$ and the geometric and material characteristics of the cantilever, as outline din Section III.

The amplitude of zero point oscillations of the cantilever is of the order of $a_{0}=\sqrt{\hbar /\left(2 m \omega_{\mathrm{r}}\right)} \approx 5 \times 10^{-13} \mathrm{~m}$, where $m \approx 6 \times 10^{-17} \mathrm{~kg}$ is the resonator mass and $\omega_{\mathrm{r}} /(2 \pi) \approx 4 \mathrm{MHz}$ is the first resonance frequency of the cantilever. Details on the cantilever are given in Section 3.

This oscillation amplitude is definitely smaller than the asymmetry imposed by the external magnetic field, i.e. $\Delta h\left(B_{0}\right) \gg a_{0}$. Nevertheless, oscillations of the cantilever produce a varying magnetic field due to the attached magnetic tips, which is proportional to the oscillation amplitude. The key consequence of the asymmetry is that constants of the interaction between the spins and the magnetic tips $\lambda_{1,2}$ are different for different spins and the Rabi transition frequencies $\Omega_{1,2}$ are different as well. Therefore, the Hamiltonian of the system of two NV spins interacting with the deformed cantilever reads

$$
\begin{aligned}
\hat{H}= & \hat{H}_{0}+\hat{V} \\
\hat{H}_{0}= & \frac{1}{2} \hbar \omega_{1} \sigma_{1}^{z}+\frac{1}{2} \hbar \omega_{2} \sigma_{2}^{z}+\hbar \omega_{r}\left(a^{+} a+1 / 2\right), \\
\hat{V}= & \lambda_{1}\left(a^{+}+a\right) \frac{1}{2} \hbar\left[\cos \theta_{1} \sigma_{1}^{z}+\sin \theta_{1}\left(\sigma_{1}^{+}+\sigma_{1}^{-}\right)\right]+ \\
& \lambda_{2}\left(a^{+}+a\right) \frac{1}{2} \hbar\left[\cos \theta_{2} \sigma_{2}^{z}+\sin \theta_{2}\left(\sigma_{2}^{+}+\sigma_{2}^{-}\right)\right] .
\end{aligned}
$$

Here the term $\hbar \omega_{\mathrm{r}}\left(a^{+} a+1 / 2\right)$ describes quantized oscillations of the cantilever, $\tan \theta_{1,2}=-\frac{\Omega_{1,2}}{\delta}$ and $\omega_{1,2}=$ $\sqrt{\Omega_{1,2}^{2}+\delta^{2}}$, thereby denotes $\Omega_{1,2}$ the Rabi frequency of the transition between up and down spin states and $\delta$ is the detuning between the microwave frequency and the intrinsic frequency of the spins. In the symmetric case $h_{1}=h_{2}=h, \omega_{1}=\omega_{2}=\omega$ and $\lambda_{1}=\lambda_{2}=\lambda$ the Hamiltonian (11) recovers the previously studied model ${ }^{24,25}$. The 
coupling constants between the spins and the cantilever has the form $\lambda_{1,2}=g_{\mathrm{S}} \mu_{\mathrm{B}} G_{1,2}^{\mathrm{m}} a_{0}$, where $g_{\mathrm{S}} \approx 2, \mu_{\mathrm{B}}$ is the Bohr magneton and $G_{1,2}^{\mathrm{m}}=\frac{1}{\hat{z}}\left|\vec{B}_{\text {tip }}\right|$ is the magnetic field gradient produced by the magnetic tips. Note that if the asymmetry of the deformation of the cantilever is strong $\Delta h\left(B_{0}\right) \sim 1$, the distance between the magnetic tip and the nearest to the cantilever adjacent NV spin becomes very small $z=h_{1}$ leading to the very large magnetic field gradient $G_{1}^{\mathrm{m}}=\frac{1}{h_{1}}\left|\vec{B}_{\mathrm{tip}}\right|$ and to the large interaction constant $\lambda_{1} \approx g_{\mathrm{S}} \mu_{\mathrm{B}} G_{1,2}^{\mathrm{m}} a_{0} / h_{1}$, while the coupling to the second spin becomes weak $\lambda_{2} \approx g_{\mathrm{S}} \mu_{\mathrm{B}} G_{1,2}^{\mathrm{m}} a_{0} / h_{2}$ and therefore the relation $\frac{\lambda_{1}}{\lambda_{2}}=\frac{h_{2}}{h_{1}} \gg 1$ holds. This means that one can easily control the interaction between the magnetic tips and the NV spins simply by tuning the amplitude of the external magnetic field and thus controlling the deflection of the cantilever $\Delta h\left(B_{0}\right)$. In the symmetric case $z=h_{1,2}=h, \Delta h=0, G_{1}^{\mathrm{m}}=G_{2}^{\mathrm{m}}=G_{\mathrm{m}} \approx 10^{6}[\mathrm{~T} / \mathrm{m}]$ and realistic values of the parameters are: $h \approx 25 \mathrm{~nm}$, $\lambda /(2 \pi) \approx 0.1[\mathrm{MHz}], \omega_{\mathrm{r}} /(2 \pi) \approx 5 \mathrm{MHz}$. In what follows the interaction constant $\lambda$ and the detuning between the cantilever frequency and the spin splitting $\Delta=\omega_{\mathrm{r}}-\omega$, $\Delta \approx 2 \lambda$ defines the time scale of the problem. A deviation of the values of the constants from the values corresponding to the symmetric case reads

$$
\begin{aligned}
& \lambda_{1,2}=\lambda\left(h_{1,2}\right)=\lambda \pm \Delta \lambda, \Delta_{1,2}=\Delta\left(h_{1,2}\right)=\Delta \pm \Delta \omega, \\
& \omega_{1,2}=\sqrt{(\Omega \pm \Delta \Omega)^{2}+\delta^{2}}=\omega \pm \Delta \omega, \Delta \omega=\frac{\Omega^{2}}{\omega}(\Delta h),(9) \\
& \Delta \lambda=\lambda \Delta h, \Delta \Omega=\Omega \Delta h .
\end{aligned}
$$

Estimates of the asymmetry parameter $\Delta h\left(B_{0}\right)$ describing a deformation of the cantilever, for realistic materials and magnetic fields, follow in the next section.

\section{MAGNETIC-FIELD-INDUCED CANTILEVER DEFLECTION}

We propose to deposit a magnetic film on the cantilever to explore alternatively magnetoelastic stress or magnetic-field induced torque to induce a controlled cantilever deflection due to an external magnetic field $B_{0}$. In the first case we exploit the tendency of a film to develop magnetoelastic stress upon magnetization, and this stress induces a curvature of the thin cantilever substrate $38-40$. In the second case we exploit the magnetic torque 41 $\vec{T}=\vec{m} \times \overrightarrow{B_{0}}$, where $\vec{m}$ is the total magnetic moment of the film, which is prepared to be oriented along the cantilever length (x-direction), and $\overrightarrow{B_{0}}$ is the applied magnetic field, oriented perpendicularly to the cantilever long axis ( $\mathrm{z}-$ direction). In this geometry a cantilever deflection along the z-direction results.

For a quantitative determination of the resulting cantilever deflection we specify the cantilever dimensions as follows: length $L=3000 \mathrm{~nm}$, width $w=300 \mathrm{~nm}$, thickness $t_{s}=30 \mathrm{~nm}$. These parameters are well suited for the nano-fabrication of Si cantilevers ${ }^{42}$. They also represent a valid scenario of stress-induced free two-dimensional bending due to the large length-to-width ratio 43 .

We assume that the cantilever is fabricated out of $\mathrm{Si}(100)$. We use the corresponding ${ }^{44}$ Young modulus $Y=$ $130 \mathrm{GPa}$ and Poisson ratio $\nu=0.279$, and density $\rho=$ $2.33 \times 10^{3} \mathrm{~kg} / \mathrm{m}^{-3}$. Such a cantilever has a mass of $m=$ $6.29 \times 10^{-17} \mathrm{~kg}$. Its first three resonance frequencies 45 are calculated from $f_{\text {res }}=t_{\mathrm{s}} \beta^{2}(Y /(3 \rho))^{0.5} /\left(4 \pi L^{2}\right)$ with $\beta=(1.8751,4.6941,7.8548)$ as $4.02,25.2$ and $70.6 \mathrm{MHz}$. This cantilever has a negligible deflection at its end due to its own weight of $3 \rho L^{4} /\left(2 Y t_{\mathrm{s}}^{2}\right)=2.4 \times 10^{-15} \mathrm{~m}$, which is more than six orders of magnitude smaller than the field induced deflection, as described next.

\section{Magnetoelastic-stress-induced cantilever deflection}

Magnetoelastic stress is responsible for the change of length of a bulk sample upon magnetization, and the resulting strain is known as magnetostriction ${ }^{38,46}$. In films, a change of length of the film upon magnetization is not possible due to the bonding to the substrate, and the film develops a magnetoelastic stress. This stress induces a curvature of the substrate, which we exploit to deflect the end of the cantilever. The role of the external magnetic field is to induce a reorientation of the magnetization of the film from an in-plane (external magnetic field off) to an out-of-plane direction (external magnetic field on along $z$-direction). Such a reorientation of the magnetization direction of the film with thickness $t_{\mathrm{f}}$ induces a corresponding change of magnetoelastic stress. It induces a deflection of the free cantilever end given by: ${ }^{38}$

$$
d e f l_{\mathrm{me}}=\frac{3 L^{2} t_{\mathrm{f}}(1+\nu) B_{1}}{Y t_{\mathrm{s}}^{2}}
$$

For a magnetization reversal along the axes of a cubic system the magnetoelastic coupling coefficient $B_{1}$ enters $^{38}$. For definiteness, we assume a Ni film of thickness $10 \mathrm{~nm}$ and we take $B_{1}$ of bulk Ni, $9.38 \mathrm{MJ} / \mathrm{m}^{3}$. We note that the effective magnetoelastic coupling in thin films may deviate from its bulk value $e^{38,39,47}$, but we stick to the bulk value for this proof of principle case study. With these assumptions we get a deflection of de $f l_{\text {me }}=27.7 \mathrm{~nm}$. A Ni film with bulk properties deposited on the top surface of the cantilever has a tendency to contract along the magnetization direction. Thus, the cantilever would be curved upwards for zero external field (magnetization in-plane along $x$ ), and it would curve downward for magnetic field on (magnetization along $z$ ). This is irrespective of the sign (along $+z$ or along $-z$ ) of the external field. For AC magnetic fields $\left(\omega_{\mathrm{mag}}\right)$ the deflection will change with $2 \omega_{\text {mag }}$.

The required deflection can be adjusted by varying the experimental parameters $t_{\mathrm{f}}, t_{\mathrm{s}}, L$, accordingly. Note that the magnitude of the external magnetic field does not enter. The only requirement is that it is large 
enough to induce a magnetization reorientation. The effective magnetic anisotropy of the magnetic film should be fairly small to achieve this. It can be tuned by adjusting film thickness, morphology, interface modifications, multilayer structures, and film composition to achieve this goal. The maximum field is given by the requirement $\mu_{\mathrm{B}} B_{0}<\hbar \omega_{0}$, as pointed out above. This gives $B_{0}<32.7 \mathrm{mT}$.

\section{Magnetic-torque-induced cantilever deflection}

The deposition of a magnetic film on the cantilever opens also the possibility to exploit the torque induced by the external magnetic field along the $z$-direction on the magnetic moments oriented along the $x$-direction of the film to induce a cantilever deflection 48 . The magnetic torque $\vec{T}=\vec{m} \times \overrightarrow{B_{0}}$ is proportional to both the total magnetic moment of the film $m$ and the external magnetic field $B_{0}$. Here, we assume that the magnetic anisotropy of the film is large enough to ensure an in-plane magnetic moment in presence of the out-of-plane field. Thus, the requirement on the magnetic anisotropy of the film differs from that discussed above for the magnetoelasticstress-induced deflection, as here an effective magnetic anisotropy favoring in-plane magnetization is required.

For definiteness we assume a magnetic moment of $2 \mu_{\mathrm{B}}$ per film atom, and we refer to the atomic volume of bulk Ni, $\rho_{\text {atomic }}=1.096 \times 10^{-29} \mathrm{~m}^{-3}$ to calculate the total number of $\mathrm{Ni}$ atoms in the film. We obtain for the torque-induced deflection ${ }^{41}$ def $l_{\text {torque }}=4 T L^{2} /\left(Y w t^{3}\right)$, and from this we find

$$
\text { def } l_{\text {torque }}=\frac{4 t_{\mathrm{f}} 2 \mu_{\mathrm{B}} B_{0} L^{3}}{\rho_{\text {atomic }} Y t_{\mathrm{s}}^{3}} .
$$

We obtain a deflection of $5.2 \mathrm{~nm}$ for the parameters quoted above at a film thickness of $t_{\mathrm{f}}=10 \mathrm{~nm}$ for an external field $B_{0}=10 \mathrm{mT}$. Note that here the deflection is proportional to the external magnetic field, which allows a continuous control of the deflection. The magnetic film could be deposited on both sides of the cantilever, which would double the deflection in a given external magnetic field. Larger deflections at a given field are obtained by e.g. increasing the film thickness.

We foresee that the exploitation of the magnetoelastic stress for achieving a well defined cantilever deflection is experimentally more challenging as compared to the exploitation of torque. One reason is the required exact tuning of the magnetic anisotropy. The torque approach is much more robust in that aspect, as only a sufficiently large energy barrier against out-of-plane magnetization is needed. Already the shape anisotropy of out-of-plane magnetization fulfills this requirement ${ }^{41}$. Therefore we focus now on the torque-induced deflection.

To quantify the indirect interaction between the NV spins mediated by the interaction with the magnetic tip we consider again the asymmetry parameter $\Delta h\left(B_{0}\right)=$ $2\left|h_{1}-h_{2}\right| /\left(h_{1}+h_{2}\right)$. We calculate the magnetic tip-NV spin distances $h_{1}, h_{2}$ as $h_{1}=h_{0}-d e f l$ and $h_{2}=h_{0}+d e f l$, where $h_{0}$ describes the symmetric case, i.e. the cantilever end is at the center position between both NV spins, which is realized for zero magnetic field for the torqueinduced deflection. This gives the asymmetry parameter

$$
\Delta h\left(B_{0}\right)=2 \frac{d e f l_{\text {torque }}}{h_{0}}=\frac{16 t_{\mathrm{f}} \mu_{\mathrm{B}} B_{0} L^{3}}{h_{0} \rho_{\text {atomic }} Y t_{\mathrm{s}}^{3}}
$$

\section{EFFECTIVE HAMILTONIAN: INDIRECT INTERACTION BETWEEN SPINS}

Interaction of the NV spins with the magnetic tips leads to an indirect interaction between the NV spins. The Hamiltonian of the indirect interaction between the NV spins can be evaluated using the Fröhlich method 35

$$
\hat{H}_{\mathrm{eff}}=\frac{i}{2} \lambda^{2} \int_{-\infty}^{0} d t^{\prime}\left[V\left(t^{\prime}\right), V(0)\right], \hat{V}(t)=e^{-i \hat{H}_{0} t} \hat{V} e^{i \hat{H}_{0} t}
$$

Taking into account eqs. (8) and (13) in the rotating wave approximation we deduce

$$
\begin{aligned}
\hat{H}_{\mathrm{eff}}= & -\frac{1}{4}\left\{\sin ^{2} \theta_{1} \frac{\lambda_{1}^{2}}{\Delta_{1}}(2 n+1) \sigma_{1}^{z}+\sin ^{2} \theta_{2} \frac{\lambda_{2}^{2}}{\Delta_{2}}(2 n+1) \sigma_{2}^{z}\right\} \\
& -\frac{1}{4} \sin \theta_{1} \sin \theta_{2}\left(\frac{\lambda_{1} \lambda_{2}}{\Delta_{1}}+\frac{\lambda_{1} \lambda_{2}}{\Delta_{2}}\right)\left(\sigma_{1}^{+} \sigma_{2}^{-}+\sigma_{1}^{-} \sigma_{2}^{+}\right), \\
n= & \left\langle\left\langle a^{+} a\right\rangle\right\rangle, \Delta_{1}=\omega_{r}-\omega_{1}, \Delta_{2}=\omega_{r}-\omega_{2} .
\end{aligned}
$$

Taking into account that $\sin \theta_{1,2} \approx \frac{\Omega}{\omega}\left(1 \pm \frac{\delta^{2}}{\omega^{2}} \Delta h\right), \frac{\delta^{2}}{\omega^{2}} \ll$ 1 and, therefore, $\sin \theta_{1,2} \approx \frac{\Omega}{\omega}=\sin \theta$ we can rewrite the interaction constant in terms of the asymmetry parameter

$$
\begin{aligned}
& \lambda_{1} \lambda_{2} \sin ^{2} \theta \frac{\Delta_{1}+\Delta_{2}}{\Delta_{1} \Delta_{2}} \approx \frac{2 \lambda^{2} \Omega^{2}}{\omega^{2} \Delta} \cdot \frac{\left(1-(\Delta h)^{2}\right)}{1-\left(\frac{\Omega^{2}}{\omega \Delta}\right)^{2}(\Delta h)^{2}}= \\
& \frac{2 \lambda^{2}}{\omega}\left(\frac{\Omega^{2}}{\omega \Delta}\right)\left[1+\left(\left(\frac{\Omega^{2}}{\omega \Delta}\right)^{2}-1\right)(\Delta h)^{2}\right] .
\end{aligned}
$$

From eq. (15) we see that the asymmetry can enhance the interaction between the spins if

$$
\frac{\Omega^{4}}{\omega^{2} \Delta^{2}}>1
$$

In the opposite case,

$$
\frac{\Omega^{4}}{\omega^{2} \Delta^{2}}<1
$$

the asymmetry lowers the interaction strength. Since the variance of parameters is relatively large $\frac{24,25}{2 \pi} \frac{\omega_{\mathrm{r}}}{2 \pi} \approx$ $1 \div 5 \mathrm{MHz}, \Omega \approx 0.1 \div 10 \mathrm{MHz}, \delta \approx 0.01 \div 0.1 \mathrm{MHz}$, both cases given by eqs. (16) and (17) can be realized. 
In particular from eq. 115) we see that the interaction strength between spins depends on the asymmetry $\Delta h$ and the dimensionless parameter $\alpha=\frac{\Omega^{2}}{\omega \Delta}$. Considering standard values of the parameters $24,25 \omega \approx \Omega, \lambda=\frac{\Delta}{2}$ from eq. (15) for the interaction strength between spins we get: $\lambda\left[1+\left(\alpha^{2}-1\right)(\Delta h)^{2}\right]$. We see that the dependence of the interaction strength between the NV spins on the asymmetry parameter $\Delta h$ is not trivial. For small values of the parameter $\alpha<1$ the asymmetry arising from the deformation of cantilever leads to the reduction of interaction strength, while for $\alpha>1$ the asymmetry increases the interaction strength. In particular, for the values $\alpha=3, \Delta h=0.5$ the interaction between $\mathrm{NV}$ spins is three times larger than the interaction strength in the symmetric case $\sim 3 \lambda$. Stronger interaction between spins means larger entanglement. Therefore controlling the spin coupling strength we can influence the degree of entanglement as well. From the physical point of view small values of the parameter $\alpha \approx \frac{\Omega}{\omega_{r}-\Omega}<1$ corresponds to a large detuning between oscillation frequency of the cantilever $\omega_{r}$ and the Rabi frequency $\Omega \mathrm{s}$, while for $\alpha>1$ we have the opposite case.

\section{INFLUENCE OF ASYMMETRY ON THE ENTANGLEMENT DEGREE}

In order to study the influence of the asymmetry on the degree of entanglement, we directly solve the Schrödinger equation corresponding to the Hamiltonian (14)

$i \frac{d|\psi\rangle}{d t}=\hat{H}_{\mathrm{eff}}|\psi\rangle$,

$|\psi\rangle=C_{1}(t)\left|\Phi^{+}\right\rangle+C_{2}(t)\left|\Phi^{-}\right\rangle+C_{3}(t)\left|\psi^{+}\right\rangle+C_{4}(t)\left|\psi^{-}\right\rangle$.

Here $\left|\Phi^{ \pm}\right\rangle$and $\left|\psi^{ \pm}\right\rangle$are Bell states ${ }^{35-37}$. Taking into account eqs. (14), (18) for the resolution coefficients we obtain

$$
\begin{aligned}
C_{1}(t)= & C_{1}(0) \cos (A t)-C_{2}(0) \sin (A t), \\
C_{2}(t)= & C_{2}(0) \cos (A t)-C_{1}(0) \sin (A t), \\
C_{3}(t)= & \frac{D^{2} C_{3}(0)-D(F+B) C_{4}(0)}{2(F+B) F} e^{i F t} \\
& +\frac{D^{2} C_{3}(0)+D(F-B) C_{4}(0)}{2(F-B) F} e^{-i F t}, \\
C_{4}(t)= & \frac{-D C_{3}(0)+(F+B) C_{4}(0)}{2 F} e^{i F t} \\
& +\frac{D C_{3}(0)+(F-B) C_{4}(0)}{2 F} e^{-i F t},
\end{aligned}
$$




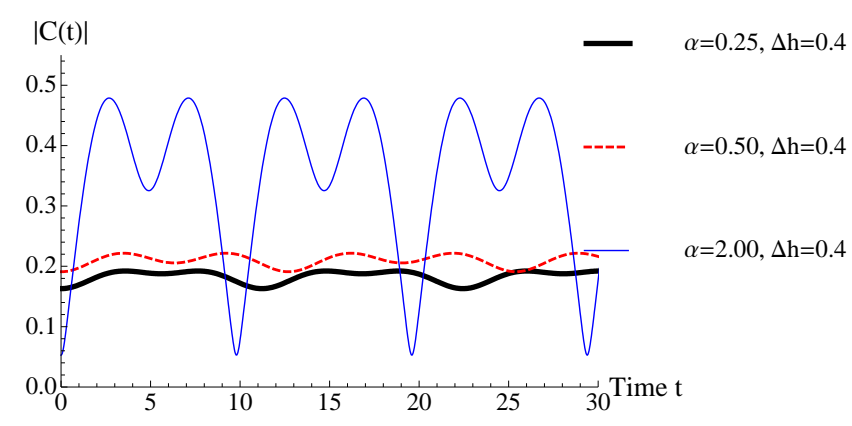

FIG. 3. Time evolution of the concurrence $|C(t)|$ for the given values of $\alpha$ and $\Delta h$. Other parameters read: $n=1$, $C_{1}=C_{2}=C_{3}=C_{4}=1 / 2$. Time-scale corresponds to the microsecond.

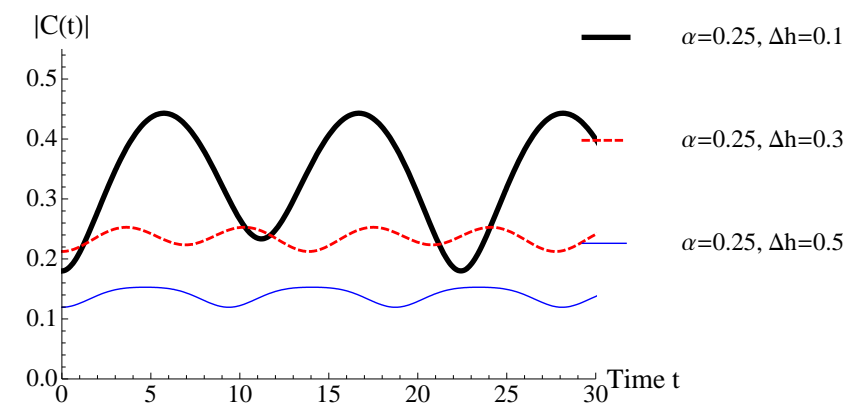

FIG. 4. Time evolution of the concurrence $|C(t)|$ for the given values of $\alpha$ and $\Delta h$. Other parameters read: $n=1$, $C_{1}=C_{2}=C_{3}=C_{4}=1 / 2$. Time-scale corresponds to the microsecond.

\section{CONCLUSIONS}

One of the main challenges for NV spin-based nanoelectromechanical resonator has been the achievement of a high controlled degree of entanglement and a strong coupling between NV spins. With this in mind, we proposed in this work a new type of nano-electromechanical resonator, the functionality of which is based on the NV spin controlled by an external magnetic field. In particular, we suggest to deposit a thin magnetic Ni film on the $\mathrm{Si}(100)$ cantilever to exploit alternatively magnetoelastic stress or magnetic-field induced torque for inducing a controlled cantilever deflection upon acting with an external magnetic field. We have shown that, depending on the values of parameter $\alpha=\frac{\Omega^{4}}{\omega^{2} \Delta^{2}}$, the induced asymmetry of the cantilever deflection substantially modifies the characteristics of the system. In particular we demonstrated that is if $\alpha>1$ the asymmetry enhances the strength of the interaction between the NV spins at least three times $\sim 3 \lambda$, where $\lambda=100 \mathrm{kHz}$ is the maximal coupling strength between NV spins for the symmetric model reported in 24 . However, for $\alpha<1$ the asymmetry reduces the interaction strength. In addition we found

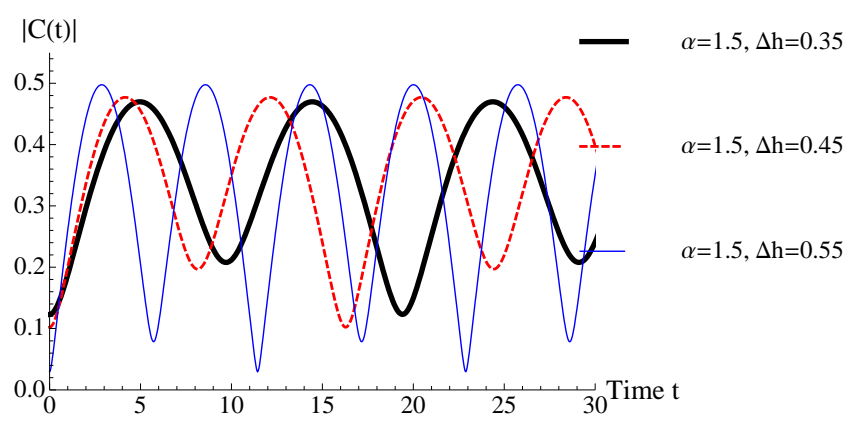

FIG. 5. Time evolution of the concurrence $|C(t)|$ for the given values of $\alpha$ and $\Delta h$. Other parameters read: $n=1$, $C_{1}=C_{2}=C_{3}=C_{4}=1 / 2$. Time-scale corresponds to the microsecond.

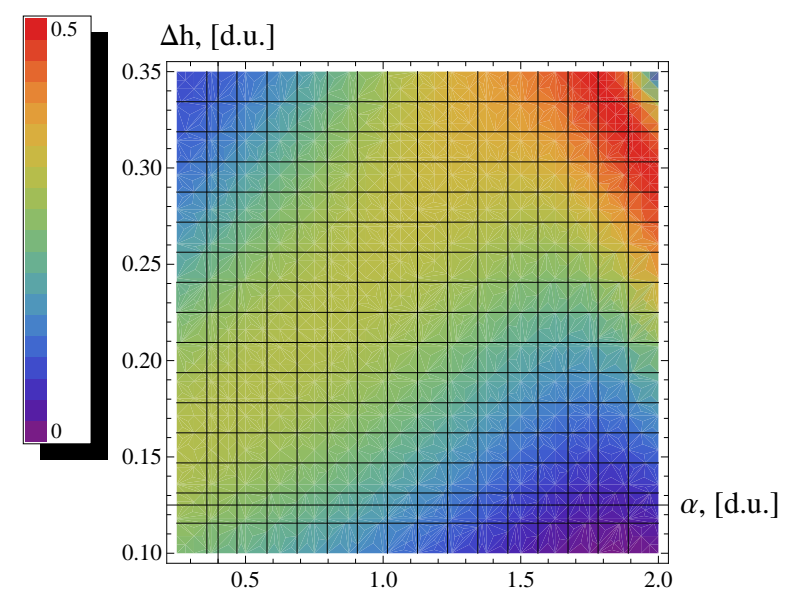

FIG. 6. Contour plot of the concurrence $|C(t)|$ for $t=13.3$. Other parameters are: $n=1, C_{1}=C_{2}=C_{3}=C_{4}=1 / 2$.

that for $\alpha>1$ entanglement is maximum for the case of a large asymmetry $\Delta h$, while for $\alpha<1$ the entanglement is maximal in the small asymmetry case (See Fig. 6). The values of the parameter $\alpha$ can be changed efficiently via the change of the detuning between the oscillation frequency of the cantilever and the spin splitting frequency $\Delta=\omega_{r}-\omega$. This can be used as an effective tool for a practical implementation of our theoretical proposal for controlling the entanglement and the interaction strength between NV spins in the experiment.

\section{ACKNOWLEDGMENTS}

The financial support by the Deutsche Forschungsgemeinschaft (DFG) through SFB 762, contract BE 2161/5-1, 15 Grant No. KO-2235/3 is gratefully acknowledged. This work is partly supported by the National Science Center in Poland as a research project in years 2011 - 2014. 
1 A. K. Naik, M. S. Hanay, W. K. Hiebert, X. L. Feng, and M. L. Roukes, Nature Nanotechnology 4, 445 - 450 (2009).

2 A. D. OConnell, M. Hofheinz, M. Ansmann, R. C. Bialczak, M. Lenander, E. Lucero, M. Neeley, D. Sank, H. Wang, and M. Weides et al., Nature (London) 464, 697 (2010).

3 A. H. Safavi-Naeini, T. P. M. Alegre, J. Chan, M. Eichenfield, M. Winger, Q. Lin, J. T. Hill, D. Chang, and O. Painter, Nature (London) 472, 69 (2011).

4 K. Stannigel, P. Rabl, A. S. Sorensen, P. Zoller, and M. D. Lukin, Phys. Rev. Lett. 105, 220501 (2010).

5 A. H. Safavi-Naeini and O. Painter, New J. Phys. 13, 013017 (2011).

6 S. Camerer, M. Korppi, A. Jöckel, D. Hunger, T.W. Hänsch, and P. Treutlein, Phys. Rev. Lett. 107, 223001 (2011).

7 M. Eichenfield, J. Chan, R. M. Camacho, K. J. Vahala, and O. Painter, Nature (London) 462, 78 (2009).

8 A. H. Safavi-Naeini et al., Phys. Rev. Lett. 108, 033602 (2012).

9 N. Brahms, T. Botter, S. Schreppler, D. W. C. Brooks, and D. M. Stamper-Kurn, Phys. Rev. Lett. 108, 133601 (2012).

10 A. Nunnenkamp, K. Borkje, and S. M. Girvin Phys. Rev. A 85, 051803 (2012)

11 F.Ya. Khalili, H. Miao, H. Yang, A.H. Safavi-Naeini, O. Painter, and Y. Chen Phys. Rev. A 86, 033840 (2012).

12 C.P. Meaney, R. H. McKenzie, and G.J. Milburn, Phys. Rev. E 83, 056202 (2011).

13 J. Atalaya, A. Isacsson, and M.I. Dykman Phys. Rev. B 83, 045419 (2011).

14 P. Rabl, Phys. Rev. B 82, 165320 (2010).

15 S.V. Prants, J. Phys. A 44, 265101 (2011).

16 M. Ludwig, K. Hammerer, and F. Marquardt, Phys. Rev. A 82, 012333 (2010).

17 T.L. Schmidt, K.B. Ørkje, C. Bruder, and B. Trauzettel, Phys. Rev. Lett. 104, 177205 (2010).

18 R.B. Karabalin, M.C. Cross, and M.L. Roukes, Phys. Rev. B 79, 165309 (2009).

19 L. Chotorlishvili, A. Ugulava, G. Mchedlishvili, A. Komnik, S. Wimberger, J. Berakdar, J. Phys. B: At. Mol. Opt. Phys. 44, 215402 (2011).

20 S.N. Shevchenko, A.N. Omelyanchouk, and E. Il'ichev, Low Temp. Phys. 38, 283 (2012).

21 Y.-X. Liu, A. Miranowicz, Y.B. Gao, J. Bajer, C.P. Sun, and F. Nori, Phys. Rev. A 82, 032101 (2010).

22 S.N. Shevchenko, S. Ashhab, F. Nori, Phys. Rep. 492, 1 (2010).

23 D. Zueco, G.M. Reuther, S. Kohler, and P. Hanggi, Phys. Rev. A 80, 033846 (2009).
24 P. Rabl, P. Cappellaro, M.V. Gurudev-Dutt, L. Jiang, J.R. Maze, and M.D. Lukin, Phys. Rev. B 79, 041302(R) (2009).

25 L.-G. Zhou, L.F. Wei, M. Gao, and X.-B. Wang, Phys. Rev. A 81, 042323 (2010).

26 O. Arcizet1, V. Jacques, A. Siria, P. Poncharal, P. Vincent, and S. Seidelin, Nat. Phys. 7, 879 (2011).

27 D. Rugar, R. Budakian, H.J. Mamin, B.W. Chui, Nature 430, 15 (2004).

28 P. Treutlein, Science 335, 1584 (2012).

29 S. Kolkowitz, A.C. Bleszynski-Jayich, Q.P. Unterreithmeier, S.D. Bennett, P. Rabl, J.G.E. Harris, M.D. Lukin, Science 335, 1603 (2012).

30 Z-H. Wang, V.V. Dobrovitski, Phys. Rev. B 84, 045303 (2011).

31 J. Wrachtrup, F. Jelezko, J. Phys.: Condens. Matter 18, S807 (2006).

32 L. Chotorlishvili, Z. Toklikishvili, Phys. Lett. A 372, 2806 (2008).

33 H. Yoo, J. Eberly, Phys. Rep. 118, 240 (1985).

34 H. Fröhlich, Proc. R. Soc. London, Ser. A 215, 291 (1952), P. Tarasewicz and D. Baran, Phys. Rev. B 73, 094524 (2006), Quantum Theory of Solids, C. Kittel, (Wiley, 1987).

35 F. Mintert, A. Carvalho, M. Kus, A. Buchleitner, Phys. Rep. 415, 207 (2005).

${ }^{36}$ L. Amico, R. Fazio, A. Osterloh, V. Vedral, Rev. Mod. Phys. 80, 517 (2008).

37 W.K. Wootters, Phys. Rev. Lett. 80, 2245 (1998).

38 D. Sander, Rep. Prog. Phys. 62, 809 (1999).

39 D. Sander, J. Phys.: Condens. Matter 16, R603 (2004).

40 D. Sander, Sensors 8, 4466 (2008).

41 Th. Höpfl, D. Sander, J. Kirschner, Rev. Sci. Instrum. 72, 1495 (2001).

42 D. Saya, K. Fukushima, H. Toshiyoshi, G. Hashiguchi, H. Fujita, H. Kawakatsu, Sensors and Actuators A 95, 281 (2002).

43 K. Dahmen, H. Ibach, D. Sander, J. Magn. Magn. Mater. 231, 74 (2001).

44 W.A. Brantley, J. Appl. Phys. 44, 534 (1973).

45 Exploring Scanning Probe Microscopy with Mathematica, D. Sarid, Wiley, 1997.

46 Ch. Kittel, Rev. Mod. Phys. 21, 541 (1949).

47 D. Sander, J. Kirschner, physica st. sol. (b) 248, 2389 (2011).

48 D. Sander, R. Skomski, C. Schmidthals, A. Enders, D. Reuter, J. Kirschner, J. Phys. D: Appl. Phys. 31, 663 (1998). 